\title{
Changes in the Angular Vestibulo-Ocular Reflex after a Single Dose of Intratympanic Gentamicin for Ménière's Disease
}

\author{
J.P. CAREY, ${ }^{a}$ T. HIRVONEN, ${ }^{a}$ G.C.Y. PENG,${ }^{b}$ C.C. DELLA SANTINA, ${ }^{a}$ \\ P. D. CREMER,${ }^{c}$ T. HASLWANTER, ${ }^{d}$ AND L.B. MINOR $^{a}$ \\ Departments of Otolaryngology ${ }^{a}$ and Neurology, ${ }^{b}$ The Johns Hopkins University \\ School of Medicine, Baltimore, Maryland 21287, USA \\ ${ }^{c}$ Eye and Ear Research Unit, Institute of Clinical Neurosciences, \\ Royal Prince Alfred Hospital, Sydney, Australia \\ ${ }^{d}$ Department of Neurology, Zürich University Hospital, Zürich, Switzerland
}

KeYwords: Ménière's disease; angular vestibulo-ocular reflex; gentamicin; intractable vertigo

\begin{abstract}
Ménière's disease is an inner ear disorder that causes episodic vertigo, hearing loss, tinnitus, and fullness in the ear. The clinical disorder has been associated with hydrops, a distension of the endolymphatic fluid compartment of the inner ear. The majority of patients can successfully control their attacks of vertigo with diuretics and restriction of sodium intake. However, about $30 \%$ of patients with Ménière's disease have intractable vertigo that does not respond to these measures. The intratympanic (middle ear) injection of gentamicin is now an alternative to ablative surgery for these patients. Gentamicin is an aminoglycoside antibiotic that is toxic to the hair cells of the inner ear, with somewhat greater vestibular toxicity than cochlear toxicity. We have treated 102 patients with unilateral Ménière's disease with intratympanic gentamicin. Attacks of vertigo have been completely controlled in $83 \%$, and substantially controlled ( $>60 \%$ reduction in frequency) in $12 \%$. Profound hearing loss occurred as a result of treatment in only $2 \%$, and the overall rate at which hearing declined after treatment (19\%) did not differ from the expected decline in hearing with active Ménière's disease.

Recent evidence suggests that a single dose of intratympanic gentamicin can be effective in controlling vertigo in Ménière's disease. ${ }^{1}$ This study was undertaken to determine the effect of a single intratympanic injection of gentamicin on the function of the human angular vestibulo-ocular reflex (AVOR) in subjects with Ménière's disease.
\end{abstract}

Address for correspondence: John P. Carey, M.D., Otolaryngology-Head and Neck Surgery, The Johns Hopkins University School of Medicine, 601 N. Caroline St., 6th floor, Baltimore, MD 21287-0910. Voice: 410-955-3403; fax: 410-955-0035.

jcarey@jhmi.edu

Ann. N.Y. Acad. Sci. 956: 581-584 (2002). () 2002 New York Academy of Sciences. 
TABLE 1. AVOR gain values for head thrusts that excited the indicated semicircular canals in subjects with Ménière's disease pre- and post-gentamicin treatment and for subjects with SUVD

\begin{tabular}{llllllll}
\hline & \multicolumn{3}{c}{ Ipsilateral canals } & & \multicolumn{3}{c}{ Contralateral canals } \\
\cline { 2 - 3 } \cline { 6 - 8 } & \multicolumn{1}{c}{ HC } & AC & \multicolumn{1}{c}{ PC } & & HC & AC & PC \\
\hline Gent-Pre & $0.94 \pm 0.18$ & $0.80 \pm 0.21$ & $0.84 \pm 0.10$ & & $0.93 \pm 0.09$ & $0.89 \pm 0.05$ & $0.78 \pm 0.18$ \\
Gent-Post & $0.39 \pm 0.11^{* *}$ & $0.41 \pm 0.11^{*}$ & $0.35 \pm 0.15^{* *}$ & & $0.83 \pm 0.08^{* *}$ & $0.86 \pm 0.11$ & $0.72 \pm 0.21$ \\
SUVD & $0.26 \pm 0.10^{*}$ & $0.24 \pm 0.10^{*}$ & $0.26 \pm 0.07$ & & $0.70 \pm 0.14^{*}$ & $0.78 \pm 0.09$ & $0.66 \pm 0.12$ \\
\hline
\end{tabular}

NotE: Figures given are mean \pm S.D. Asterisks indicate the significance of the difference of the given mean from the one in the row above it: $* * p \leq 0.001 ; * p \leq 0.01$.

The three-dimensional AVOR responses elicited by rapid rotary head thrusts were studied in 12 subjects with unilateral Ménière's disease before and 2 to 10 weeks after a single treatment with intratympanic gentamicin and in 13 subjects after surgical unilateral vestibular destruction (SUVD). Each head thrust was in the horizontal plane or in either diagonal plane of the vertical semicircular canals (left anterior/ right posterior [LARP] or right anterior/left posterior [RALP] planes). Thus, each head thrust effectively stimulated only one pair of canals. The AVOR gains (eye velocity/head velocity during the $30 \mathrm{msec}$ before peak head velocity) for the head thrusts exciting each individual canal were averaged and taken as a measure of the function of that canal.

Before treatment with intratympanic gentamicin, subjects with unilateral Ménière's disease usually had AVOR gains similar to values reported in normal subjects (FIG. 1, TABLE 1). ${ }^{2-5}$ There were minimal asymmetries between responses for head thrusts expected to excite canals on the ipsi- versus contralateral sides (TABLE 1). Responses after a single intratympanic gentamicin injection demonstrated marked gain reductions for head thrusts expected to excite canals on the treated side (FIG. 1). On average, gains for excitation of the treated horizontal canal (HC) decreased by $59 \%$, for the treated anterior canal (AC) by $49 \%$, and for the treated posterior canal (PC) by 58\% (T ABLE 1). Gains for head thrusts that excited the contralateral canals decreased by much smaller percentages: $\mathrm{HC}, 11 \%$; $\mathrm{AC}, 4 \%$; and $\mathrm{PC}$, $8 \%$. In comparisons of the gains of the subjects with Ménière's disease after treatment with intratympanic gentamicin and the gains of the SUVD subjects, the gains were lower for the SUVD subjects for those head thrusts that excited the HC and AC on the lesioned (ipsilateral) side and the $\mathrm{HC}$ on the contralateral side. These significant differences are indicated by asterisks in TABLE 1.

Ménière's disease itself did not reduce the AVOR gain values for the affected semicircular canals in most of our subjects with the disorder. Tsuji et al. examined temporal bones from 24 patients with longstanding Ménière's disease. ${ }^{6}$ While the loss of type II hair cells and cells of Scarpa's ganglion were greater than expected for age, they rarely observed $>50 \%$ reduction in either type of hair cell or their afferent neurons. Our data suggest that the preservation of these structures is adequate to preserve AVOR function in response to rapid rotary head thrusts in subjects with symptomatic Ménière's disease.

In the cases presented here, a single treatment with intratympanic gentamicin controlled the attacks of vertigo due to Ménière's disease during the initial 

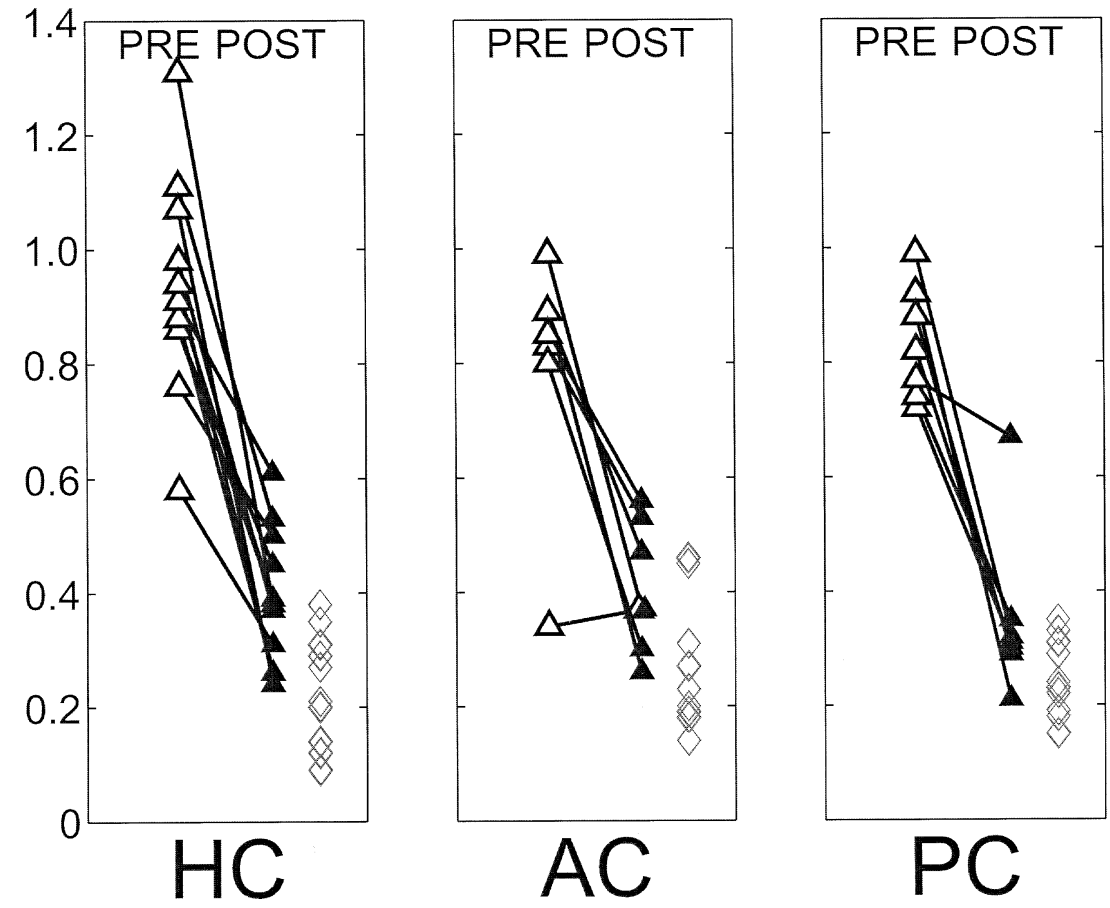

FIGURE 1. Comparison of gain values measured in 10 subjects with unilateral Ménière's disease for head thrusts that excited the semicircular canals on the affected side before intratympanic gentamicin treatment (PRE) and following treatment (POST). Triangles represent mean gain values from individual subjects with Ménière's disease, and each line connects pre- and post-gentamicin values for one subject. Filled triangles in the POST data indicate decreased gains compared to PRE values $(p<0.01)$. The open diamonds show gain values for head thrusts toward the lesioned side in SUVD subjects.

(6 months) follow-up period. In every case the single dose of gentamicin markedly reduced AVOR gains for the semicircular canals on the treated side. The effect of intratympanic gentamicin was specific to the treated ear, as indicated by the more modest reduction in AVOR gains for excitation of canals on the contralateral side. The gain reduction for the ipsilateral horizontal and anterior canals caused by intratympanic gentamicin treatment was not as great as that caused by SUVD.

These results suggest that a single treatment with intratympanic gentamicin might not cause complete hair cell destruction. Indeed, partial hair cell loss in the labyrinth has been noted in the chinchilla after a single intraotic application of gentamicin. ${ }^{7,8}$ The hair cells remaining after intratympanic gentamicin treatment may provide some ipsilateral excitatory component to the response that is missing in the SUVD subjects. An alternative or adjunctive mechanism for the improved response after intratympanic gentamicin may be the preservation of baseline afferent discharge on the treated side. Such preservation of the afferent firing would maintain the symmetry in afferent activity reaching the central vestibular nuclei. During a rap- 
id head thrust exciting the canals on the treated side, the balanced central vestibular activity might improve the response generated by inhibition of afferents from the contralateral side.

\section{ACKNOWLEDGMENTS}

This work was supported by NIDCD Grants K23 DC00196 and R01 DC02390.

\section{REFERENCES}

1. Harner, S.G. et al. 2001. Long-term follow-up of transtympanic gentamicin for Ménière's syndrome. Otol. Neurotol. 22: 210-214.

2. Aw, S.T. et al. 1996. Three-dimensional vector analysis of the human vestibulo-ocular reflex in response to high-acceleration head rotations. I. Responses in normal subjects. J. Neurophysiol. 76: 4009-4020.

3. CRemer, P.D. et al. 1998. Semicircular canal plane head impulses detect absent function of individual semicircular canals. Brain 121: 699-716.

4. TABAK, S. et al. 1997. Gain and delay of human vestibulo-ocular reflexes to oscillation and steps of the head by a reactive torque helmet. I. Normal subjects. Acta Otolaryngol. (Stockh.) 117: 785-795.

5. Tian, J.-R. et al. 2001. Impairments of the initial horizontal vestibulo-ocular reflex in older humans. Exp. Brain Res. 137: 309-322.

6. TsusI, K. et al. 2000. Temporal bone studies of the human peripheral vestibular system. 4. Ménière's disease. Ann. Otol. Rhinol. Laryngol. 109: 26-31.

7. LOPEZ, I. et al. 1997. Quantification of the process of hair cell loss and recovery after gentamicin treatment. Int. J. Dev. Neurosci. 15: 447-461.

8. LOPEZ, I. et al. 1998. Hair cell recovery in the chinchilla crista ampullaris after gentamicin treatment: a quantitative approach. Otolaryngol. Head Neck Surg. 119: 255-262. 